\title{
Strings and Multi-Strings in Black Hole and Cosmological Spacetimes ${ }^{1}$
}

\author{
A.L. Larsen and N. Sánchez \\ Observatoire de Paris, DEMIRM. Laboratoire Associé au CNRS \\ UA 336, Observatoire de Paris et École Normale Supérieure. \\ 61, Avenue de l'Observatoire, 75014 Paris, France.
}

February 4, 2020

\footnotetext{
${ }^{1}$ Research report to appear in "New developments in string gravity and physics at the Planck energy scale". Edited by N. Sánchez (World Scientific, Singapore, 1995)
} 


\begin{abstract}
Recent results on classical and quantum strings in a variety of black hole and cosmological spacetimes, in various dimensions, are presented. The curved backgrounds under consideration include the $2+1$ black hole anti de Sitter spacetime and its dual, the black string, the ordinary $D \geq 4$ black holes with or without a cosmological constant, the de Sitter and anti de Sitter spacetimes and static Robertson-Walker spacetimes. Exact solutions to the string equations of motion and constraints, representing circular strings, stationary open strings and dynamical straight strings, are obtained in these backgrounds and their physical properties (length, energy, pressure) are described. The existence of multi-string solutions, describing finitely or infinitely many strings, is shown to be a general feature of spacetimes with a positive or negative cosmological constant. Generic approximative solutions are obtained using the string perturbation series approach, and the question of the stability of the solutions is addressed.

Furthermore, using a canonical quantization procedure, we find the string mass spectrum in de Sitter and anti de Sitter spacetimes. New features as compared to the string spectrum in flat Minkowski spacetime appear, for instance the fine-structure effect at all levels beyond the graviton in both de Sitter and anti de Sitter spacetimes, and the non-existence of a Hagedorn temperature in anti de Sitter spacetime. We discuss the physical implications of these results. Finally, we consider the effect of spatial curvature on the string dynamics in Robertson-Walker spacetimes.
\end{abstract}




\section{Introduction}

The classical and quantum propagation of strings in curved spacetimes has attracted a great deal of interest in recent years. The main complication, as compared to the case of flat Minkowski spacetime, is related to the nonlinearity of the equations of motion. It makes it possible to obtain the complete analytic solution only in a very few special cases like conical spacetime [1] and plane-wave/shock-wave backgrounds [2]. There are however also very general results concerning integrability and solvability for maximally symmetric spacetimes [3, 4] and gauged WZW models [5, 6]. These are the exceptional cases; generally the string equations of motion in curved spacetimes are not integrable and even if they are, it is usually an extremely difficult task to actually separate the equations, integrate them and finally write down the complete solution in closed form. Fortunately, there are several different ways to "attack" a system of coupled non-linear partial differential equations.

The systematic study of string dynamics in curved spacetimes and its associated physical phenomena was started in Refs. [7, 8]. Besides numerical methods, which will not be discussed here, approximative [7-10] and exact [11-14] methods for solving the string equations of motion and constraints in curved spacetimes, have been developed. Classical and quantum string dynamics have been investigated in black hole backgrounds [15-18], cosmological spacetimes [7, 11-14, 17-21], cosmic string spacetime [1], gravitational wave backgrounds [2], supergravity backgrounds (which are necessary for fermionic strings) [22], and near spacetime singularities [23]. Physical phenomena like the Hawking-Unruh effect in string theory [8, 24, horizon string stretching [8, 24], particle transmutation [15, 25], string scattering [1, 2, 15], mass spectrum and critical dimension [1, 7, 15, 27], string instability [7, 11$13,16,21]$ and multi-string solutions [11-13, 28] have been found.

In a generic $D$-dimensional curved spacetime with metric $g_{\mu \nu}$ and coordinates $x^{\mu},(\mu=0,1, \ldots, D-1)$, the string equations of motion and constraints are:

$$
\begin{aligned}
& \ddot{x}^{\mu}-x^{\prime \mu}+\Gamma_{\rho \sigma}^{\mu}\left(\dot{x}^{\rho} \dot{x}^{\sigma}-x^{\prime \rho} x^{\prime \sigma}\right)=0, \\
& g_{\mu \nu} \dot{x}^{\mu} x^{\prime \nu}=g_{\mu \nu}\left(\dot{x}^{\mu} \dot{x}^{\nu}+x^{\prime \mu} x^{\prime \nu}\right)=0,
\end{aligned}
$$

where dot and prime stand for derivative with respect to the world-sheet coordinates $\tau$ and $\sigma$, respectively and $\Gamma_{\rho \sigma}^{\mu}$ are the Christoffel symbols with respect to the metric $g_{\mu \nu}$. In the following we present recent results [26-29] 
on solutions to Eqs.(1.1)-(1.2) in a variety of curved spacetimes from cosmology, gravitation and string theory. We obtain explicit (exact and/or approximate) mathematical solutions, discuss the corresponding physical properties, we quantize the solutions in different ways (canonical quantization, semiclassical quantization) and find the physical content: the mass spectrum.

The presentation is organized as follows: In Section 2, we discuss the classical string dynamics in the $2+1$ black hole anti de Sitter (BH-AdS) spacetime, recently found by Bañados et al [30]. We compare with the string dynamics in ordinary cosmological and black hole spacetimes. This clarifies the geometry (as seen by a string) of the BH-AdS spacetime. In Section 3 , generalizing results from Section 2, we derive the quantum string mass spectrum in ordinary $D$-dimensional anti de Sitter spacetime. We discuss, in particular, the sectors of low and very high mass states. New physical phenomena arise like the fine-structure effect at all levels beyond the graviton and the non-existence of a Hagedorn critical temperature. The results are compared with corresponding results obtained in Minkowski and de Sitter spacetimes. Sections 4 and 5 are devoted to the investigation of the more general underlying structure of solutions to Eqs.(1.1)-(1.2). In Section 4, we find new classes of exact string and multi-string solutions in cosmological and black hole spacetimes, while in Section 5, we consider the effects of a non-zero spatial curvature on the classical and quantum string dynamics in Friedmann-Robertson-Walker (FRW) universes.

\section{Classical String Dynamics in $2+1$ BH-AdS}

Anti de Sitter (AdS) spacetime is often considered to be a spacetime of minor importance in a cosmological context. However, first of all, it is a FRW universe and as such should not be neglected, secondly; it serves as a simple and convenient spacetime for comparison and understanding of results obtained in (say) Minkowski or de Sitter spacetimes and third, it has a tendency to show up (in disguise) as a solution in various models of dilaton-gravity and string theory. An example of the latter is represented by the $2+1 \mathrm{BH}-\mathrm{AdS}$ spacetime of Bañados et al [30]. This spacetime background has arised much interest recently [31-35]. It describes a two-parameter family (mass $M$ and angular momentum $J$ ) of black holes in $2+1$ dimensional general relativity 
with metric:

$$
d s^{2}=\left(M-\frac{r^{2}}{l^{2}}\right) d t^{2}+\left(\frac{r^{2}}{l^{2}}-M+\frac{J^{2}}{4 r^{2}}\right)^{-1} d r^{2}-J d t d \phi+r^{2} d \phi^{2} .
$$

It has two horizons $r_{ \pm}=\sqrt{\frac{M l^{2}}{2} \pm \frac{l}{2} \sqrt{M^{2} l^{2}-J^{2}}}$ and a static limit $r_{\mathrm{erg}}=$ $\sqrt{M} l$, defining an ergosphere, as for ordinary Kerr black holes. The spacetime is not asymptotically flat; it approaches anti de Sitter spacetime asymptotically with cosmological constant $\Lambda=-1 / l^{2}$. The curvature is constant $R_{\mu \nu}=-\left(2 / l^{2}\right) g_{\mu \nu}$ everywhere, except probably at $r=0$, where it has at most a delta-function singularity. Notice the weak nature of the singularity at $r=0$ in $2+1$ dimensions as compared with the power law divergence of curvature scalars in $D>3$ (We will not discuss here the geometry near $r=0$. For a discusion, see Refs. 33, 35]). The spacetime, Eq.(2.1), is also a solution of the low energy effective action of string theory with zero dilaton field $\Phi=0$, anti-symmetric tensor field $H_{\mu \nu \rho}=\left(2 / l^{2}\right) \epsilon_{\mu \nu \rho} \quad$ (i.e. $\left.B_{\phi t}=r^{2} / l^{2}\right)$ and $k=l^{2}$ 31. Moreover, it yields an exact solution of string theory in $2+1$ dimensions, obtained by gauging the WZWN sigma model of the group $S L(2, R) \times R$ at level $k$ [31, 32 (for non-compact groups, $k$ does not need to be an integer, so the central charge $c=3 k /(k-2)=26$ when $\mathrm{k}=52 / 23)$. This solution is the black string background [36]:

$$
\begin{gathered}
d \tilde{s}^{2}=-\left(1-\frac{\mathcal{M}}{\tilde{r}}\right) d \tilde{t}^{2}+\left(1-\frac{\mathcal{Q}^{2}}{\mathcal{M} \tilde{r}}\right) d \tilde{x}^{2}+\left(1-\frac{\mathcal{M}}{\tilde{r}}\right)^{-1}\left(1-\frac{\mathcal{Q}^{2}}{\mathcal{M} \tilde{r}}\right)^{-1} \frac{l^{2} d \tilde{r}^{2}}{4 \tilde{r}^{2}}, \\
\tilde{B}_{\tilde{x} \tilde{t}}=\frac{\mathcal{Q}}{r}, \quad \tilde{\Phi}=-\frac{1}{2} \log \tilde{r} l
\end{gathered}
$$

which is related by duality [31, 37] to the 2+1 BH-AdS spacetime, Eq.(2.1). It has two horizons $\tilde{r}_{ \pm}=r_{ \pm}$, the same as the metric, Eq.(2.1), while the static limit is $\tilde{r}_{\text {erg }}=J /(2 \sqrt{M})$.

We first investigate the string propagation in the $2+1$ BH-AdS background by considering the perturbation series around the exact center of mass of the string:

$$
x^{\mu}(\tau, \sigma)=q^{\mu}(\tau)+\eta^{\mu}(\tau, \sigma)+\xi^{\mu}(\tau, \sigma)+\ldots
$$

The original method of Refs. [7, 8] can be conveniently formulated in covariant form. It is useful to introduce $D-1$ normal vectors $n_{R}^{\mu}(R=1, . ., D-1)$, 
(which can be chosen to be covariantly constant by gauge fixing), and consider comoving perturbations $\delta x_{R}$, i.e. those seen by an observer travelling with the center of mass, thus $\eta^{\mu}=\delta x^{R} n_{R}^{\mu}$. After Fourier expansion, $\delta x^{R}(\tau, \sigma)=\sum_{n} C_{n}^{R}(\tau) e^{-i n \sigma}$, the first order perturbations satisfy the matrix Schrödinger-type equation in $\tau$ :

$$
\ddot{C}_{n R}+\left(n^{2} \delta_{R S}-R_{\mu \rho \sigma \nu} n_{R}^{\mu} n_{S}^{\nu} \dot{q}^{\rho} \dot{q}^{\sigma}\right) C_{n}^{S}=0 .
$$

Second order perturbations $\xi^{\mu}$ and constraints are similarly covariantly treated, $\xi^{\mu}$ also satisfying Schrödinger-type equations with source terms, see Ref.[26].

For our purposes here it is enough to consider the non-rotating $(J=0)$ $2+1 \mathrm{BH}-\mathrm{AdS}$ background and a radially infalling string. We have solved completely the c.m. motion $q^{\mu}(\tau)$ and the first and second order perturbations $\eta^{\mu}(\tau, \sigma)$ and $\xi^{\mu}(\tau, \sigma)$ in this background. Eqs.(2.4) become:

$$
\ddot{C}_{n R}+\left(n^{2}+\frac{m^{2}}{l^{2}}\right) C_{n R}=0 ; \quad R=\perp, \|
$$

The first order perturbations are independent of the black hole mass, only the anti de Sitter (AdS) part emerges. All oscillation frequencies $\omega_{n}=$ $\sqrt{n^{2}+m^{2} / l^{2}}$ are real; there are no unstable modes in this case. The perturbations:

$$
\delta x_{R}(\tau, \sigma)=\sum_{n}\left[A_{n R} e^{-i\left(n \sigma+\omega_{n} \tau\right)}+\tilde{A}_{n R} e^{-i\left(n \sigma-\omega_{n} \tau\right)}\right]
$$

are completely finite and regular. This is also true for the second order perturbations which are bounded everywhere, even for $r \rightarrow 0(\tau \rightarrow 0)$. We have also computed the conformal generators $L_{n}$, (see Ref. [26]), and the string mass:

$$
m^{2}=2 \sum_{n}\left(2 n^{2}+\frac{m^{2}}{l^{2}}\right)\left[A_{n \|} \tilde{A}_{-n \|}+A_{n \perp} \tilde{A}_{-n \perp}\right]
$$

The mass formula is modified (by the term $\mathrm{m}^{2} / l^{2}$ ) with respect to the usual flat spacetime expression. This is due to the asymptotic (here AdS) behaviour of the spacetime. In ordinary $D \geq 4$ black hole spacetimes (without cosmological constant), which are asymptotically flat, the mass spectrum is the same as in flat Minkowski spacetime 15. 
We compare with the string perturbations in the ordinary $(D \geq 4)$ black hole anti de Sitter spacetime. In this case Eqs.(2.4) become:

$$
\begin{gathered}
\ddot{C}_{n S \perp}+\left(n^{2}+m^{2} H^{2}+\frac{M m^{2}}{r^{3}}\right) C_{n S \perp}=0, \quad S=1,2 \\
\ddot{C}_{n \|}+\left(n^{2}+m^{2} H^{2}-\frac{2 M m^{2}}{r^{3}}\right) C_{n \|}=0 .
\end{gathered}
$$

The transverse $\perp$-perturbations are oscillating with real frequencies and are bounded even for $r \rightarrow 0$. For longitudinal $\|$-perturbations, however, imaginary frequencies arise and instabilities develop. The $(|n|=1)$-instability sets in at:

$$
r_{\text {inst. }}=\left(\frac{2 M m^{2}}{1+m^{2} H^{2}}\right)^{1 / 3}
$$

Lower modes become unstable even outside the horizon, while higher modes develop instabilities at smaller $r$ and eventually only for $r \approx 0$. For $r \rightarrow 0$ (which implies $\tau \rightarrow \tau_{0}$ ) we find $r(\tau) \approx(3 m \sqrt{M / 2})^{2 / 3}\left(\tau_{0}-\tau\right)^{2 / 3}$ and:

$$
\begin{gathered}
\ddot{C}_{n S \perp}+\frac{2}{9\left(\tau-\tau_{0}\right)^{2}} C_{n S \perp}=0, \quad S=1,2 \\
\ddot{C}_{n \|}-\frac{4}{9\left(\tau-\tau_{0}\right)^{2}} C_{n \|}=0 .
\end{gathered}
$$

For $\tau \rightarrow \tau_{0}$ the $\|$-perturbations blow up while the string ends trapped into the $r=0$ singularity. We see the important difference between the string evolution in the $2+1 \mathrm{BH}-\mathrm{AdS}$ background and the ordinary $3+1$ (or higher dimensional) black hole anti de Sitter spacetime.

We also compare with the string propagation in the $2+1$ black string background, Eq.(2.2) (with $J=0$ ). In this case, Eqs.(2.4) become:

$$
\begin{gathered}
\ddot{C}_{n \perp}+n^{2} C_{n \perp}=0, \\
\ddot{C}_{n \|}+\left(n^{2}-\frac{2 m^{2} M}{l r}\right) C_{n \|}=0 .
\end{gathered}
$$

The $\perp$-modes are stable, while $C_{n \|}$ develop instabilities. For $r \rightarrow 0$ (which implies $\left.\tau \rightarrow \tau_{0}\right)$ we find $r(\tau) \approx \frac{m^{2} M}{l}\left(\tau_{0}-\tau\right)^{2}$ and:

$$
\ddot{C}_{n \|}-\frac{2}{\left(\tau_{0}-\tau\right)^{2}} C_{n \|}=0,
$$


with similar conclusions as for the ordinary $3+1$ (or higher dimensional) black hole anti de Sitter spacetime.

In order to extract more information about the string evolution in these backgrounds, in particular exact properties, we consider the circular string ansatz:

$$
t=t(\tau), \quad r=r(\tau), \quad \phi=\sigma+f(\tau),
$$

in the equatorial plane $(\theta=\pi / 2)$ of the stationary axially symmetric backgrounds:

$$
d s^{2}=g_{t t}(r) d t^{2}+g_{r r}(r) d r^{2}+2 g_{t \phi}(r) d t d \phi+g_{\phi \phi}(r) d \phi^{2} .
$$

This includes all the cases of interest here: The $2+1 \mathrm{BH}-\mathrm{AdS}$ spacetime, the black string, as well as the equatorial plane of ordinary Einstein black holes. The string dynamics, determined by Eqs.(1.1)-(1.2), is then reduced to a system of second order ordinary differential equations and constraints, also described as a Hamiltonian system:

$$
\begin{gathered}
\dot{r}^{2}+V(r)=0 ; \quad V(r)=g^{r r}\left(g_{\phi \phi}+E^{2} g^{t t}\right), \\
\dot{t}=-E g^{t t}, \quad \dot{f}=-E g^{t \phi} ; \quad E=-P_{t}=\text { const. }
\end{gathered}
$$

which in all backgrounds considered here are solved in terms of either elementary or elliptic functions. The dynamics of the circular strings takes place at the $r$-axis in the $(r, V(r))$-diagram and from the properties of the potential $V(r)$ (minima, zeroes, asymptotic behaviour for large $r$ and the value $V(0)$ ), general knowledge about the string motion can be obtained. On the other hand, the line element of the circular string turns out to be:

$$
d s^{2}=g_{\phi \phi}\left(d \sigma^{2}-d \tau^{2}\right), \quad \text { i.e. } S(\tau)=\sqrt{g_{\phi \phi}(r(\tau))},
$$

$S(\tau)$ being the invariant string size. For all the static black hole AdS spacetimes ( $2+1$ and higher dimensional): $S(\tau)=r(\tau)$.

For the rotating $2+1 \mathrm{BH}-\mathrm{AdS}$ spacetime:

$$
V(r)=r^{2}\left(\frac{r^{2}}{l^{2}}-M\right)+\frac{J^{2}}{4}-E^{2},
$$

(see Fig.1). $V(r)$ has a global minimum $V_{\text {min }}<0$ between the two horizons $r_{+}, r_{-}$(for $M l^{2} \geq J^{2}$, otherwise there are no horizons). The vanishing of 
$V(r)$ at $r=r_{01,2}$ (see Ref.[26]) determines three different types of solutions: (i) For $J^{2}>4 E^{2}$, there are two positive zeroes $r_{01}<r_{02}$, the string never comes outside the static limit, never falls into $r=0$ neither (there is a barrier between $r=r_{01}$ and $r=0$ ). The mathematical solution oscillates between $r_{01}$ and $r_{02}$ with $0<r_{01}<r_{-}<r_{+}<r_{02}<r_{\text {erg. }}$. It may be interpreted as a string travelling between the different universes described by the maximal analytic extension of the manifold. (ii) For $J^{2}<4 E^{2}$, there is only one positive zero $r_{0}$ outside the static limit and there is no barrier preventing the string from collapsing into $r=0$. The string starts at $\tau=0$ with maximal size $S_{\max }^{(i i)}$ outside the static limit, it then contracts through the ergosphere and the two horizons and eventually collapses into a point $r=0$. For $J \neq 0$, it may be still possible to continue this solution into another universe like in the case (i). (iii) $J^{2}=4 E^{2}$ is the limiting case where the maximal string size equals the static limit: $S_{\max }^{(i i i)}=l \sqrt{M}$. In this case $V(0)=0$, thus the string contracts through the two horizons and eventually collapses into a point $r=0$.

The exact general solution in the three cases (i)-(iii) is given by:

$$
r(\tau)=\left|r_{m}-\frac{1}{c_{1} \wp\left(\tau-\tau_{0}\right)+c_{2}}\right|,
$$

where:

$$
r_{m}=S_{\max }=\sqrt{\frac{M l^{2}}{2}} \sqrt{1+\sqrt{1-\frac{4 V(0)}{M l^{2}}}}, \quad V(0)=\frac{J^{2}}{4}-E^{2} .
$$

$c_{1}, c_{2}$ are constants in terms of $\left(l, M, r_{m}\right)$, given in Ref.[26], and $\wp$ is the Weierstrass elliptic $\wp$-function with invariants $\left(g_{2}, g_{3}\right)$, discriminant $\Delta$ and roots $\left(e_{1}, e_{2}, e_{3}\right)$, also given in Ref. [26]. The three cases (i)-(iii) correspond to the cases $\Delta>0, \Delta<0$ and $\Delta=0$, respectively. Notice that $S_{\max }^{(i i)}>$ $S_{\max }^{(i i i)}=l \sqrt{M}>S_{\max }^{(i)}$. In the case (i), $r(\tau)$ can be written in terms of the Jacobian elliptic function $\operatorname{sn}\left[\tau^{*}, k\right], \tau^{*}=\sqrt{e_{1}-e_{3}} \tau, k=\sqrt{\left(e_{2}-e_{3}\right) /\left(e_{1}-e_{3}\right)}$. It follows that the solution (i) oscillates between the two zeroes $r_{01}$ and $r_{02}$ of $V(r)$, with period $2 \omega$, where $\omega$ is the real semi-period of the Weierstrass function: $\omega=K(k) / \sqrt{e_{1}-e_{3}}$, in terms of the complete elliptic integral of the first kind $K(k)$. We have:

$$
r(0)=r_{m}, \quad r(\omega)=\sqrt{M l^{2}-r_{m}^{2}}, \quad r(2 \omega)=r_{m}, \ldots
$$


In the case (ii) $(\Delta<0)$ two roots $\left(e_{1}, e_{3}\right)$ become complex, the string collapses into a point $r=0$ and we have:

$$
r(0)=r_{m}, \quad r\left(\frac{\omega_{2}}{2}\right)=0, \quad r\left(\omega_{2}\right)=r_{m}, \ldots
$$

where $\omega_{2}$ is the real semi-period of the Weierstrass function for this case. In the case (iii) $(\Delta=0)$ the elliptic functions reduce to hyperbolic functions:

$$
r(\tau)=\frac{\sqrt{M} l}{\cosh (\sqrt{M} \tau)},
$$

so that:

$$
r(-\infty)=0, \quad r(0)=r_{m}=\sqrt{M} l, \quad r(+\infty)=0 .
$$

Here, the string starts as a point, grows until $r=r_{m}$ (at $\tau=0$ ), and then it contracts until it collapses again $(r=0)$ at $\tau=+\infty$. In this case the string makes only one oscillation between $r=0$ and $r=r_{m}$.

Notice that for the static background $(J=0)$, the only allowed motion is (ii), i.e. $r_{m}>r_{\text {hor }}=\sqrt{M} l$ (there is no ergosphere and only one horizon in this case), with:

$$
r_{m}=\sqrt{\frac{M l^{2}}{2}} \sqrt{1+\sqrt{1+\frac{4 E^{2}}{M l^{2}}}} .
$$

For $J=0$, the string collapses into $r=0$ and stops there. The Penrose diagram of the $2+1 \mathrm{BH}$-AdS spacetime for $J=0$ is very similar to the Penrose diagram of the ordinary $(D \geq 4)$ Schwarzschild spacetime, so the string motion outwards from $r=0$ is unphysical because of the causal structure. The coordinate time $t(\tau)$ can be expressed in terms of the incomplete elliptic integral of the third kind $\Pi$, see Ref. [26]. The string has its maximal size $r_{m}$ at $\tau=0$, passes the horizon at $\tau=\tau_{\text {hor }}$ (expressed in terms of an incomplete elliptic integral of the first kind) and falls into $r=0$ for $\tau=\omega_{2} / 2, \omega_{2}$ being the real semi-period of the Weierstrass function. That is, we have:

$$
\begin{gathered}
r(0)=r_{m}, \quad r\left(\tau_{\text {hor }}\right)=\sqrt{M} l, \quad r\left(\omega_{2} / 2\right)=0, \\
t(0)=0, \quad t\left(\tau_{\text {hor }}\right)=\infty
\end{gathered}
$$

and $t\left(\omega_{2} / 2\right)$ can be expressed in terms of the Jacobian zeta function $Z$, see Ref. 26. 
We also study the circular strings in the ordinary $D \geq 4$ spacetimes. In the generic $3+1$ Kerr anti de Sitter (or Kerr de Sitter) spacetime, the potential $V(r)$ is however quite complicated, see Ref. 26]. The general circular string solution involves higher genus elliptic functions and it is not necessary to go into details here. We will compare with the non-rotating cases, only.

It is instructive to recall [38 the circular string in Minkowski spacetime (Min), for which $V(r)=r^{2}-E^{2}$ (Fig.2a), the string oscillates between its maximal size $r_{m}=E$, and $r=0$ with the solution $r(\tau)=r_{m}|\cos \tau|$.

In the Schwarzschild black hole (S) $V(r)=r^{2}-2 M r-E^{2}$ (Fig.2b), the solution is remarkably simple: $r(\tau)=M+\sqrt{M^{2}+E^{2}} \cos \tau$. The mathematical solution oscillates between $r_{m}=M+\sqrt{M^{2}+E^{2}}$ and $M-\sqrt{M^{2}+E^{2}}<0$, but because of the causal structure and the curvature singularity the motion can not be continued after the string has collapsed into $r=0$.

For anti de Sitter spacetime (AdS), we find: $V(r)=r^{2}\left(1+H^{2} r^{2}\right)-E^{2}$ (Fig.2c). The string oscillates between $r_{m}=\frac{1}{\sqrt{2} H} \sqrt{-1+\sqrt{1+4 H^{2} E^{2}}}$ and $r=0$ with the solution:

$$
r(\tau)=r_{m}\left|\operatorname{cn}\left[\left(1+4 H^{2} E^{2}\right)^{1 / 4}, k\right]\right|,
$$

which is periodic with period $2 \omega$ :

$$
\omega=\frac{K(k)}{\left(1+4 H^{2} E^{2}\right)^{1 / 4}}, \quad k=\sqrt{\frac{\sqrt{1+4 H^{2} E^{2}}-1}{2 \sqrt{1+4 H^{2} E^{2}}}} .
$$

For Schwarzschild anti de Sitter spacetime (S-AdS), we find $V(r)=r^{2}(1+$ $\left.H^{2} r^{2}\right)-2 M r-E^{2}$ (Fig.2d) and:

$$
r(\tau)=r_{m}-\frac{1}{d_{1 \wp(\tau)+d_{2}}}, \quad r(0)=r_{m}
$$

$d_{1}, d_{2}$ are constants given in terms of $\left(M, H, r_{m}\right)$, see Ref. 26] $\left(r_{m}\right.$ is the root of the equation $V(r)=0$, which has in this case exactly one positive solution). The invariants, the discriminant and the roots are also given explicitly in Ref. 26]. The string starts with $r=r_{m}$ at $\tau=0$, it then contracts and eventually collapses into the $r=0$ singularity. The existence of elliptic function solutions for the string motion is characteristic of the presence of a cosmological constant. For $\Lambda=0=-3 H^{2}$ the circular string motion reduces to simple trigonometric functions. From Fig.2 and our analysis we 
see that the circular string motion is qualitatively very similar in all these backgrounds (Min, S, AdS, S-AdS): the string has a maximal bounded size and then it contracts towards $r=0$. There are however physical and quantitative differences: in Minkowski and pure anti de Sitter spacetimes, the string truly oscillates between $r_{m}$ and $r=0$, while in the black hole cases (S, S-AdS), there is only one half oscillation, the string motion stops at $r=0$. This also holds for the $2+1 \mathrm{BH}$-AdS spacetime with $J=0$ (Fig.1b). Notice also that in all these cases, $V(0)=-E^{2}<0$ and $V(r) \sim r^{\alpha} ;(\alpha=2,4)$ for $r>>E$.

The similarity can be pushed one step further by considering small perturbations around the circular strings. We find [18]:

$$
\ddot{C}_{n}+\left(n^{2}+\frac{r}{2} \frac{d a(r)}{d r}+\frac{r^{2}}{2} \frac{d^{2} a(r)}{d r^{2}}-\frac{2 E^{2}}{r^{2}}\right) C_{n}=0,
$$

determining the Fourier components of the comoving perturbations. For the spacetimes of interest here, $a(r)=1-2 M / r+H^{2} r^{2}$ (Min, S, AdS, S-AdS), or $a(r)=r^{2} / l^{2}-1(2+1 \mathrm{BH}-\mathrm{AdS})$, the comoving perturbations are regular except near $r=0$, where we find (for all cases) $r(\tau) \approx-E\left(\tau-\tau_{0}\right)$ and:

$$
\ddot{C}_{n}-\frac{2}{\left(\tau-\tau_{0}\right)^{2}} C_{n}=0
$$

It follows that not only the unperturbed circular strings, but also the comoving perturbations around them behave in a similar way in all these non-rotating backgrounds $(2+1$ and higher dimensional). This should be contrasted with the string perturbations around the center of mass, which behave differently in these backgrounds. It must be noticed that for rotating $(J \neq 0)$ spacetimes, the circular string behaviour is qualitatively different from the non-rotating $(J=0)$ spacetimes. For large $J$, both in the $2+1$ $\mathrm{BH}-\mathrm{AdS}$ as well as in the $3+1$ ordinary Kerr-AdS spacetimes, non-collapsing circular string solutions exist. The potential $V(r) \rightarrow+\infty$ for $r \rightarrow 0$ and no collapse into $r=0$ is possible.

The dynamics of circular strings in curved spacetimes is determined by the string tension, which tends to contract the string, and by the local gravity (which may be attractive or repulsive). In all the previous non-rotating backgrounds, the local gravity is attractive (i.e. $d a(r) / d r>0$ ), and it acts together with the string tension in the sense of contraction. But in spacetimes with regions in which repulsion (i.e. $d a(r) / d r<0$ ) dominates, the strings can 
expand with unbounded radius (unstable strings [11, 13]). It may also happen that the string tension and the local gravity be of the same order, i.e. the two opposite effects can balance, and the string is stationary. De Sitter spacetime provides an example in which all such type of solutions exist [11, 13]. In de Sitter spacetime, $V(r)$ is unbounded from below for $r \rightarrow \infty\left(V(r) \sim-r^{4}\right)$ and unbounded expanding circular strings are present. In addition, an interesting new feature appears in the presence of a positive cosmological constant: the existence of multi-string solutions [9-11]. The world-sheet time $\tau$ turns out to be a multi (finite or infinite) valued function of the physical time. That is, one single world-sheet where $-\infty \leq \tau \leq+\infty$, can describe many (even infinitely many [13]) different and independent strings (in flat spacetime, one single world-sheet describes only one string). In the S, AdS and S-AdS spacetimes, the multi-string feature for circular strings is absent.

The main conclusions of this section are given in Tables 1, 2. Further details can be found in Ref. 26]

\section{Quantum String Spectrum in Ordinary AdS}

In the first part of the previous section, the classical string dynamics was studied in the $2+1 \mathrm{BH}$-AdS spacetime. In this section we go further in the investigation of the physical properties of strings in AdS spacetime, by performing string quantization. Since the $2+1 \mathrm{BH}-\mathrm{AdS}$ spacetime is locally AdS, the results of Section 2 can be extended to classical strings in $D$-dimensional AdS spacetime as well. In AdS spacetime, the string motion is oscillatory in time and is stable; all fluctuations around the string center of mass are well behaved and bounded. Local gravity of AdS spacetime is always negative and string instabilities do not develop. The string perturbation series approach, considering fluctuations around the center of mass, is particularly appropriate in AdS spacetime, the natural dimensionless expansion parameter being $\lambda=\alpha^{\prime} / l^{2}>0$, where $\alpha^{\prime}$ is the string tension and the 'Hubble constant' $H=1 / l$. The negative cosmological constant of AdS spacetime is related to the 'Hubble constant' $H$ by $\Lambda=-(D-1)(D-2) H^{2} / 2$.

All the spatial $(\mu=1, \ldots, D-1)$ modes in $D$-dimensional AdS, oscillate with frequency $\omega_{n}=\sqrt{n^{2}+m^{2} \alpha^{\prime} \lambda}$, which are real for all $n$ ( $m$ being the string mass). In this section, we perform a canonical quantization procedure. From the conformal generators $L_{n}, \tilde{L}_{n}$ and the constraints $L_{0}=\tilde{L}_{0}=0$, 
imposed at the quantum level, we obtain the mass formula (the quantum version of Eq.(2.7) written in units where $\alpha^{\prime}$ appears explicitly):

$$
m^{2} \alpha^{\prime}=(D-1) \sum_{n>0} \Omega_{n}(\lambda)+\sum_{n>0} \Omega_{n}(\lambda) \sum_{R=1}^{D-1}\left[\left(\alpha_{n}^{R}\right)^{\dagger} \alpha_{n}^{R}+\left(\tilde{\alpha}_{n}^{R}\right)^{\dagger} \tilde{\alpha}_{n}^{R}\right],
$$

where:

$$
\Omega_{n}(\lambda)=\frac{2 n^{2}+m^{2} \alpha^{\prime} \lambda}{\sqrt{n^{2}+m^{2} \alpha^{\prime} \lambda}}
$$

and we have applied symmetric ordering of the operators. The operators $\alpha_{n}^{R}, \tilde{\alpha}_{n}^{R}$ satisfy:

$$
\left[\alpha_{n}^{R},\left(\alpha_{n}^{R}\right)^{\dagger}\right]=\left[\tilde{\alpha}_{n}^{R},\left(\tilde{\alpha}_{n}^{R}\right)^{\dagger}\right]=1, \quad \text { for all } n>0
$$

For $\lambda<<1$, which is clearly fulfilled in most interesting cases, we have found the lower mass states $m^{2} \alpha^{\prime} \lambda<<1$ and the quantum mass spectrum [27]. Physical states are characterized by the eigenvalue of the number operator:

$$
N=\frac{1}{2} \sum_{n>0} n \sum_{R=1}^{D-1}\left[\left(\alpha_{n}^{R}\right)^{\dagger} \alpha_{n}^{R}+\left(\tilde{\alpha}_{n}^{R}\right)^{\dagger} \tilde{\alpha}_{n}^{R}\right]
$$

and the ground state is defined by:

$$
\alpha_{n}^{R}\left|0>=\tilde{\alpha}_{n}^{R}\right| 0>=0, \quad \text { for all } n>0 .
$$

We find that $m^{2} \alpha^{\prime}=0$ is an exact solution of the mass formula in $D=25$ and that there is a graviton at $D=25$, which indicates, as in de Sitter space [0], that the critical dimension of AdS is 25 (although it should be stressed that the question whether these spacetimes are solutions to the full $\beta$-function equations remains open). As in Minkowski spacetime, the ground state is a tachyon. Remarkably enough, for $N \geq 2$ we find that a generic feature of all excited states beyond the graviton, is the presence of a fine structure effect: for a given eigenvalue $N \geq 2$, the corresponding states have different masses. For the lower mass states the expectation value of the mass operator in the corresponding states (generically labelled $\mid j>$ ) turns out to have the form (see Ref.27):

$$
<j\left|m^{2} \alpha^{\prime}\right| j>_{\mathrm{AdS}}=a_{j}+b_{j} \lambda^{2}+c_{j} \lambda^{3}+\mathcal{O}\left(\lambda^{4}\right) .
$$


The collective index "j" generically labels the state $\mid j>$ and the coefficients $a_{j}, b_{j}, c_{j}$ are all well computed numbers, different for each state, their precise values are given in Ref. [27]. The corrections to the mass in Minkowski spacetime appear to order $\lambda^{2}$. Therefore, the leading Regge trajectory for the lower mass states is:

$$
J=2+\frac{1}{2} m^{2} \alpha^{\prime}+\mathcal{O}\left(\lambda^{2}\right) .
$$

In Minkowski spacetime the mass and number operator of the string are related by $m^{2} \alpha^{\prime}=-4+4 N$. In AdS (as well as in de Sitter (dS)), there is no such simple relation between the mass and the number operators; the splitting of levels increases considerably for very large $N$. The fine structure effect we find here is also present in de Sitter space. Up to order $\lambda^{2}$, the lower mass states in $\mathrm{dS}$ and in AdS are the same, the differences appear to the order $\lambda^{3}$. The lower mass states in de Sitter spacetime are given by Eq.(3.6) but with the $\lambda^{3}$-term getting an opposite sign $\left(-c_{j} \lambda^{3}\right)$.

For the very high mass spectrum, we find more drastic effects. States with very large eigenvalue $N$, namely $N>>1 / \lambda$, have masses:

$$
<j\left|m^{2} \alpha^{\prime}\right| j>_{\mathrm{AdS}} \approx d_{j} \lambda N^{2}
$$

and angular momentum:

$$
J^{2} \approx \frac{1}{\lambda} m^{2} \alpha^{\prime},
$$

where $d_{j}$ are well computed numbers different for each state, their precise values are given in Ref.[27]. Since $\lambda=\alpha^{\prime} / l^{2}$, we see from Eq.(3.8) that the masses of the high mass states are independent of $\alpha^{\prime}$. In Minkowski spacetime, very large $N$ states all have the same mass $m^{2} \alpha^{\prime} \approx 4 N$, but here in AdS the masses of the high mass states with the same eigenvalue $N$ are different by factors $d_{j}$. In addition, because of the fine structure effect, states with different $N$ can get mixed up. For high mass states, the level spacing grows with $N$ (instead of being constant as in Minkowski spacetime). As a consequence, the density of states $\rho(m)$ as a function of mass grows like $\operatorname{Exp}\left[(m / \sqrt{|\Lambda|})^{1 / 2}\right]$ (instead of $\operatorname{Exp}\left[m \sqrt{\alpha^{\prime}}\right]$ as in Minkowski spacetime), and independently of $\alpha^{\prime}$. The partition function for a gas of strings at a temperature $\beta^{-1}$ in AdS spacetime is well defined for all finite temperatures $\beta^{-1}$, discarding the existence of the Hagedorn temperature. 
For the low mass states $\left(m^{2} \alpha^{\prime} \lambda<<1\right)$ in anti de Sitter spacetime, our results can be written as [27]:

$$
<j\left|m^{2}\left(\alpha^{\prime}, l\right)\right| j>=\frac{4(N-1)}{\alpha^{\prime}}+\frac{1}{l^{2}} \sum_{n=0}^{\infty} a_{j n}\left(\alpha^{\prime} / l^{2}\right)^{n},
$$

where " $j "$ is a collective index labelling the state $\mid j>$. It is now important to notice that $a_{j 0}=0$ for all the low mass states [27], i.e. there is no "constant" term on the right hand side of Eq.(3.10). A non-zero $a_{j 0}$-term would give rise to a $\alpha^{\prime}$-independent contribution to the string mass. Its absense, on the other hand, means that the first term on the right hand side of Eq.(3.10) is super-dominant (since, in all cases, $\alpha^{\prime} / l^{2}=\lambda<<1$ ) and that the string scale is therefore set by $1 / \alpha^{\prime}$.

For the high mass states $\left(m^{2} \alpha^{\prime} \lambda>>1\right)$ we found instead [27]:

$$
<j\left|m^{2}\left(\alpha^{\prime}, l\right)\right| j>\approx \frac{d_{j}}{l^{2}} N^{2}, \quad \text { for } N>>l^{2} / \alpha^{\prime}
$$

where the number $d_{j}$ depends on the state. The right hand side of Eq.(3.11) is exactly like a non-zero dominant $a_{j 0}$-term in Eq.(3.10). For the high mass states the scale is therefore set by $1 / l^{2}$ which is equal to the absolute value of the cosmological constant $\Lambda$ (up to a geometrical factor) and independent of $\alpha^{\prime}$. This suggests that for $\lambda<<1$, the masses of all string states can be represented by a formula of the form (3.10). For the low mass states $a_{j 0}=0$, while for the high mass states $a_{j 0}$ becomes a large positive number.

In the black string background, Eq.(2.2), we have calculated explicitly the first and second order string fluctuations around the center of mass [27]. We then determined the world-sheet energy-momentum tensor and we derived the mass formula in the asymptotic region. The mass spectrum is equal to the mass spectrum in flat Minkowski spacetime. Therefore, for a gas of strings at temperature $\beta^{-1}$ in the asymptotic region of the black string background, the partition function goes like:

$$
Z(\beta) \sim \int^{\infty} d m e^{-m\left(\beta-\sqrt{\alpha^{\prime}}\right)}
$$

which is only defined for $\beta>\sqrt{\alpha^{\prime}}$, i.e. there is a Hagedorn temperature:

$$
T_{\mathrm{Hg}}=\left(\alpha^{\prime}\right)^{-1 / 2} \text {. }
$$


In higher dimensional $(D \geq 4)$ black hole spacetimes the next step now would be to set up a scattering formalism, where a string from an asymptotic instate interacts with the gravitational field of the black hole and reappears in an asymptotic out-state [15]. However, this is not possible in the black string background. In the uncharged black string background under consideration here, all null and timelike geodesics incoming from spatial infinity pass through the horizon and fall into the physical singularity [36]. No "angular momentum", as in the case of scattering off the ordinary Schwarzschild black hole, can prevent a point particle from falling into the singularity. The string solutions considered here are based on perturbations around the string center of mass which follows, at least approximately, a point particle geodesic. Therefore, a string incoming from spatial infinity inevitably falls into the singularity in the black string background.

\section{New Classes of Exact String and Multi- String Solutions in Curved Spacetimes}

In this section we return to the string equations of motion and constraints, Eqs.(1.1)-(1.2), to look for new classes of exact solutions. In most spacetimes, quite general families of exact solutions can be found by making an appropriate ansatz, which exploits the symmetries of the underlying curved spacetime. In axially symmetric spacetimes, a convenient ansatz corresponds to circular strings, as we saw in Section 2. Such an ansatz effectively decouples the dependence on the spatial world-sheet coordinate $\sigma$, and the string equations of motion and constraints reduce to non-linear coupled ordinary differential equations, Eqs.(2.18)-(2.19). In this section we will make instead an ansatz which effectively decouples the dependence on the temporal worldsheet coordinate $\tau$. This ansatz, which we call the "stationary string ansatz" is dual to the "circular string ansatz" in the sense that it corresponds to a formal interchange of the world-sheet coordinates $(\tau, \sigma)$, as well as of the azimuthal angle $\phi$ and the stationary time $t$ in the target space:

$$
\tau \leftrightarrow \sigma, \quad t \leftrightarrow \phi
$$

The stationary string ansatz will describe stationary strings when $t$ (and $\tau$ ) are timelike, for instance in anti de Sitter spacetime (in static coordinates) 
and outside the horizon of a Schwarzschild black hole. On the other hand, if $t$ (and $\tau$ ) are spacelike, for instance inside the horizon of a Schwarzschild black hole or outside the horizon of de Sitter spacetime (in static coordinates), the stationary string ansatz will describe dynamical propagating strings. Considering for simplicity a static line element in the form:

$$
d s^{2}=-a(r) d t^{2}+\frac{d r^{2}}{a(r)}+r^{2}\left(d \theta^{2}+\sin ^{2} \theta d \phi^{2}\right)
$$

the stationary string ansatz reads explicitly:

$$
t=\tau, \quad r=r(\sigma), \quad \phi=\phi(\sigma), \quad \theta=\pi / 2 .
$$

The string equations of motion and constraints, Eqs.(1.1)-(1.2), reduce to two separated first order ordinary differential equations:

$$
\phi^{\prime}=\frac{L}{r^{2}}, \quad r^{\prime 2}+V(r)=0 ; \quad V(r)=-a(r)\left[a(r)-\frac{L^{2}}{r^{2}}\right]
$$

where $L$ is an integration constant. The qualitative features of the possible string configurations can be read off directly from the shape of the potential $V(r)$. Thereafter, the detailed analysis of the quantitative features can be performed by explicitly solving the (integrable) system of equations (4.4). The induced line element on the world-sheet is given by:

$$
d s^{2}=a(r)\left(-d \tau^{2}+d \sigma^{2}\right) .
$$

Thus, if $a(r)$ is negative, the world-sheet coordinate $\tau$ becomes spacelike while $\sigma$ becomes timelike and the stationary string ansatz (4.3) describes a dynamical string. If $a(r)$ is positive, the ansatz describes a stationary string.

In this section we solve explicitly Eqs.(4.4) and we analyze in detail the solutions and their physical interpretation in Minkowski, de Sitter, anti de Sitter, Schwarzschild and $2+1$ black hole anti de Sitter spacetimes. In all these cases, the solutions are expressed in terms of elliptic (or elementary) functions. We furthermore analyze the physical properties, energy and pressure, of these solutions. The energy and pressure densities of the strings can be obtained from the $3+1$ dimensional spacetime energy-momentum tensor:

$$
\sqrt{-g} T^{\mu \nu}=\frac{1}{2 \pi \alpha^{\prime}} \int d \tau d \sigma\left(\dot{X}^{\mu} \dot{X}^{\nu}-X^{\prime \mu} X^{\prime \nu}\right) \delta^{(4)}(X-X(\tau, \sigma)) .
$$


After integration over a spatial volume that completely encloses the string [39], the energy-momentum tensor takes the form of a fluid:

$$
T_{\nu}^{\mu}=\operatorname{diag} .\left(-E, P_{1}, P_{2}, P_{3}\right) \text {. }
$$

In Minkowski spacetime (Min), the potential defined in Eqs.(4.4) is given by:

$$
V_{\mathrm{M}}(r)=\frac{L^{2}}{r^{2}}-1
$$

and the solution of Eqs.(4.4) describes one infinitely long straight string with "impact-parameter" $L$. The equation of state, relating energy and pressure densities, takes the well-known form Ref. [28]:

$$
d E=-d P_{2}=\text { const., } \quad P_{1}=P_{3}=0 .
$$

In anti de Sitter spacetime (AdS), the potential is given by:

$$
V_{\mathrm{AdS}}(r)=-\left(1+H^{2} r^{2}\right)\left[1+H^{2} r^{2}-\frac{L^{2}}{r^{2}}\right]
$$

The radial coordinate $r(\sigma)$ is periodic with finite period $T_{\sigma}$, which can be expressed in terms of a complete elliptic integral, see Ref. [28]. For $\sigma \in\left[0, T_{\sigma}\right]$, the solution describes an infinitely long stationary string in the wedge $\phi \in$ ] $0, \Delta \phi[$, where:

$$
\left.\Delta \phi=2 k \sqrt{\frac{1-2 k^{2}}{1-k^{2}}}\left[\Pi\left(1-k^{2}, k\right)-K(k)\right] \in\right] 0, \pi[
$$

The elliptic modulus $k$, which is a function of $H L$ [28], parametrizes the solutions, $k \in] 0,1 / \sqrt{2}[$. The azimuthal angle is generally not a periodic function of $\sigma$, thus when the spacelike world-sheet coordinate $\sigma$ runs through the range ] $-\infty,+\infty$ [, the solution describes an infinite number of infinitely long stationary open strings. The general solution is therefore a multi-string solution. Until now multi-string solutions were only found in de Sitter spacetime [11-13]. Our results show that multi-string solutions are a general feature of spacetimes with a cosmological constant (positive or negative). The solution in anti de Sitter spacetime describes a finite number of strings if the following relation holds:

$$
N \Delta \phi=2 \pi M
$$


Here $N$ and $M$ are integers, determining the number of strings and the winding in azimuthal angle, respectively, for the multi-string solution, see Fig.3. The equation of state for a full multi-string solution takes the form $\left(P_{3}=0\right)$ :

$$
d P_{1}=d P_{2}=-\frac{1}{2} d E, \quad \text { for } r \rightarrow \infty
$$

corresponding to extremely unstable strings [21].

In de Sitter spacetime $(\mathrm{d} S)$, the potential is given by:

$$
V_{\mathrm{dS}}(r)=-\left(1-H^{2} r^{2}\right)\left[1-H^{2} r^{2}-\frac{L^{2}}{r^{2}}\right] .
$$

In this case we have to distinguish between solutions inside the horizon (where $\tau$ is timelike) and solutions outside the horizon (where $\tau$ is spacelike). Inside the horizon, the generic solution describes one infinitely long open stationary string winding around $r=0$. For special values of the constants of motion, corresponding to a relation, which formally takes the same form as Eq.(4.12), the solution describes a closed string of finite length $l=N \pi / H$. The integer $N$ in this case determines the number of "leaves", see Fig.4. The energy is positive and finite and grows with $N$. The pressure turns out to vanish identically, thus the equation of state corresponds to cold matter.

Outside the horizon, the world-sheet coordinate $\tau$ becomes spacelike while $\sigma$ becomes timelike, thus we define:

$$
\tilde{\tau} \equiv \sigma, \quad \tilde{\sigma} \equiv \tau,
$$

and the string solution is conveniently expressed in hyperboloid coordinates. The radial coordinate $r(\tilde{\tau})$ is periodic with a finite period $T_{\tilde{\tau}}$. For $\tilde{\tau} \in\left[0, T_{\tilde{\tau}}\right]$, the solution describes a straight string incoming non-radially from spatial infinity, scattering at the horizon and escaping towards infinity again, Fig.5. The string length is zero at the horizon and grows indefinetely in the asymptotic regions. As in the case of anti de Sitter spacetime, the azimuthal angle is generally not a periodic function, thus when the timelike world-sheet coordinate $\tilde{\tau}$ runs through the range $]-\infty,+\infty[$, the solution describes an infinite number of dynamical straight strings scattering at the horizon at different angles. The general solution is therefore a multi-string solution. In particular, a multi-string solution describing a finite number of strings is obtained if a relation of the form (4.12) is fulfilled. It turns out that the 
solution describes at least three strings. The energy and pressures of a full multi-string solution have also been computed. In the asymptotic region they fulfill an equation of state corresponding to extremely unstable strings, i.e. like Eq.(4.13).

In the Schwarzschild black hole background (S), the potential is given by:

$$
V_{\mathrm{S}}(r)=-(1-2 m / r)\left[1-2 m / r-\frac{L^{2}}{r^{2}}\right] .
$$

No multi-string solutions are found in this case. Outside the horizon the solution of Eqs.(4.4) describes one infinitely long stationary open string. This solution was already derived in Ref.[40], and we shall not discuss it here. Inside the horizon, where $\tau$ becomes spacelike while $\sigma$ becomes timelike, we make the redefinitions (4.15) and the solution is conveniently expressed in terms of Kruskal coordinates, see Ref. [28]. The solution describes one straight string infalling non-radially towards the singularity, see Fig.6. At the horizon, the string length is zero and it grows indefinitely when the string approaches the spacetime singularity. Thereafter, the solution can not be continued, see Ref. [28].

In the $2+1$ black hole anti de Sitter spacetime (BH-AdS) [30], the potential is given by:

$$
V_{\mathrm{BH}-\mathrm{AdS}}(r)=-\left(\frac{r^{2}}{l^{2}}-1\right)\left[\frac{r^{2}}{l^{2}}-1-\frac{L^{2}}{r^{2}}\right] .
$$

Outside the horizon, the solutions "interpolate" between the solutions found in anti de Sitter spacetime and outside the horizon of the Schwarzschild black hole. The solutions thus describe infinitely long stationary open strings. As in anti de Sitter spacetime, the general solution is a multi-string describing infinitely many strings. In particular, for certain values of the constants of motion, corresponding to the condition of the form (4.12), the solution describes a finite number of strings. In the simplest version of the $2+1 \mathrm{BH}-\mathrm{AdS}$ background $(M=1, J=0)$, it turns out that the solution describes at least seven strings, see Ref.[28]. Inside the horizon, we make the redefinitions (4.15) and the solution is conveniently expressed in terms of Kruskal-like coordinates, see Ref.[28]. The solution is similar to the solution found inside the horizon of the Schwarzschild black hole, but there is one important difference at $r=0$. As in the Schwarzschild black hole background, the solution describes one straight string infalling non-radially towards $r=0$, and beyond 
this point the solution can not be continued because of the global structure of the spacetime. At the horizon the string size is zero and during the fall towards $r=0$, the string size grows but stays finite. This should be compared with the straight string inside the horizon of the Schwarzschild black hole, where the string size grows indefinetely. The physical reason for this difference is that the point $r=0$ is not a strong curvature singularity in the $2+1 \mathrm{BH}$-AdS spacetime.

We have considered also the Schwarzschild de Sitter and Schwarzschild anti de Sitter spacetimes. These spacetimes contain all the features of the spacetimes already discussed: singularities, horizons, positive or negative cosmological constants. All the various types of string solutions found in the other spacetimes (open, closed, straight, finitely and infinitely long, multistrings) are therefore present in the different regions of the Schwarzschild-de Sitter and Schwarzschild-anti de Sitter spacetimes. The details are given in Ref. 28.

We close this section with a few remarks on the stability of the solutions. Generally, the question of stability must be addressed by considering small perturbations around the exact solutions. In Ref. 10], a covariant formalism describing physical perturbations propagating along an arbitrary string configuration embedded in an arbitrary curved spacetime, was developed. The resulting equations determining the evolution of the perturbations are however very complicated in the general case, although partial (analytical) results have been obtained in special cases for de Sitter [18, 43] and Schwarzschild black hole 110, 43, 44 spacetimes. The exact solutions found in this section fall essentially into two classes: dynamical and stationary. The dynamical string solutions outside the horizon of de Sitter (or S-dS) and inside the horizon of Schwarzschild (or S-dS, S-AdS) spacetimes, are already unstable at the zeroth order approximation (i.e. without including small perturbations), in the sense that their physical length grows indefinetely. For the stationary string solutions the situation is more delicate. The existence of the stationary configurations is based on an exact balance between the string tension and the local attractive or repulsive gravity. For that reason, it can be expected that the configurations are actually unstable for certain modes of perturbation, especially in strong curvature regions.

The main conclusions of this section are given in Table 4. Further details can be found in Ref. 228. 


\section{Spatial Curvature Effects on the String Dy- namics}

The propagation of strings in Friedmann-Robertson-Walker (FRW) cosmologies has been investigated using both exact and approximative methods, see for example Refs.[7, 11-14, 17-20] (as well as numerical methods, which shall not be discussed here). Except for anti de Sitter spacetime, which has negative spatial curvature, the cosmologies that have been considered until now, have all been spatially flat. In this section we will consider the physical effects of a non-zero (positive or negative) curvature index on the classical and quantum strings. The non-vanishing components of the Riemann tensor for the generic D-dimensional FRW line element, in comoving coordinates:

$$
d s^{2}=-d t^{2}+a^{2}(t) \frac{d \vec{x} d \vec{x}}{\left(1+\frac{K}{4} \vec{x} \vec{x}\right)^{2}}
$$

are given by:

$$
R_{i t i t}=\frac{-a a_{t t}}{\left(1+\frac{K}{4} \vec{x} \vec{x}\right)^{2}}, \quad R_{i j i j}=\frac{a^{2}\left(K+a_{t}^{2}\right)}{\left(1+\frac{K}{4} \vec{x} \vec{x}\right)^{4}} ; \quad i \neq j
$$

where $a=a(t)$ is the scale factor and $K$ is the curvature index. Clearly, a non-zero curvature index introduces a non-zero spacetime curvature; the exceptional case provided by $K=-a_{t}^{2}=$ const., corresponds to the MilneUniverse. From Eqs.(5.2), it is also seen that the curvature index has to compete with the first derivative of the scale factor. The effects of the curvature index are therefore most conveniently discussed in the family of FRWuniverses with constant scale factor, the so-called static Robertson-Walker spacetimes. This is the point of view we take in the present section.

We consider both the closed $(K>0)$ and the hyperbolic $(K<0)$ static Robertson-Walker spacetimes, and all our results are compared with the already known results in the flat $(K=0)$ Minkowski spacetime. We determine the evolution of circular strings, derive the corresponding equations of state

(using Eq.(4.6)), discuss the question of strings as self-consistent solutions to the Einstein equations [20], and we perform a semi-classical quantization. We also find all the stationary string configurations in these spacetimes.

The radius of a classical circular string in the spacetime (5.1), for $a=1$, is 
determined by:

$$
\dot{r}^{2}+V(r)=0 ; \quad V(r)=\left(1-K r^{2}\right)\left(r^{2}-b \alpha^{\prime 2}\right),
$$

where $b$ is an integration constant $\left(b \alpha^{2}=E\right.$, in the notation of Eqs.(2.18)(2.19)). This equation is solved in terms of elliptic functions and all solutions describe oscillating strings (Fig.7 shows the potential $V(r)$ for $K>0, K=$ $0, K<0)$.

For $K>0$, when the spatial section is a hypersphere, the string either oscillates on one hemisphere or on the full hypersphere. The energy is always positive while the average pressure can be positive, negative or zero, depending on the precise value of an elliptic modulus; the equation of state is given explicitly in Table 5, in the different cases. Interestingly enough, we find that the circular strings provide a self-consistent solution to the Einstein equations with a selected value of the curvature index. Self-consistent solutions to the Einstein equations with string sources have been found previously in the form of power law expanding universes [20].

We have semi-classically quantized the circular string solutions of Eq.(5.3). We used an approach developed in field theory by Dashen et. al. 41, based on the stationary phase approximation of the partition function. The result of the stationary phase integration is expressed in terms of the function:

$$
W(m) \equiv S_{\mathrm{cl}}(T(m))+m T(m)
$$

where $S_{\mathrm{cl}}$ is the action of the classical solution, $m$ is the mass and the period $T(m)$ is implicitly given by:

$$
\frac{d S_{\mathrm{cl}}}{d T}=-m
$$

The bound state quantization condition then becomes 41]:

$$
W(m)=2 \pi n, \quad n \in N_{0}
$$

for $n$ 'large'. Parametric plots of $K \alpha^{\prime} W$ as a function of $K \alpha^{\prime 2} m^{2}$ in the different cases, are shown in Fig.8. The strings oscillating on one hemisphere give rise to a finite number $N_{-}$of states with the following mass-formula Ref. 29]:

$$
m_{-}^{2} \alpha^{\prime} \approx \pi n, \quad N_{-} \approx \frac{4}{\pi K \alpha^{\prime}}
$$


As in flat Minkowski spacetime, the scale of these string states is set by $\alpha^{\prime}$. The strings oscillating on the full hypersphere give rise to an infinity of more and more massive states with the asymptotic mass-formula Ref. [29]:

$$
m_{+}^{2} \approx K n^{2} .
$$

The masses of these states are independent of $\alpha^{\prime}$, the scale is set by the curvature index $K$. Notice also that the level spacing grows with $n$. A similar result was found recently for strings in anti de Sitter spacetime 42.

For $K<0$, when the spatial section is a hyperboloid, both the energy and the average pressure of the oscillating strings are positive. The equation of state is given in Table 5. In this case, the strings can not provide a selfconsistent solution to the Einstein equations. After semi-classical quantization, we find an infinity of more and more massive states. The mass-formula is given by (see Ref. 29]):

$$
\sqrt{-K m^{2} \alpha^{\prime 2}} \log \sqrt{-K m^{2} \alpha^{\prime 2}} \approx-\frac{\pi}{2} K \alpha^{\prime} n
$$

Notice that the level spacing grows faster than in Minkowski spacetime but slower than in the closed static Robertson-Walker spacetime.

On the other hand, the stationary strings are determined by Eqs.(4.4):

$$
\phi^{\prime}=\frac{L}{r^{2}}, \quad r^{2}+V(r)=0 ; \quad V(r)=\left(1-K r^{2}\right)\left(\frac{L^{2}}{r^{2}}-1\right),
$$

where $L$ is an integration constant (Fig.9 shows the potential $V(r)$ for $K>$ $0, K=0, K<0)$.

For $K>0$, all the stationary string solutions describe circular strings winding around the hypersphere. The equation of state is of the extremely unstable string type [21]. For $K<0$, the stationary strings are represented by infinitely long open configurations with an angle between the two "arms" given by:

$$
\Delta \phi=\pi-2 \arctan (\sqrt{-K} L) .
$$

The energy density is positive while the pressure densities are negative. No simple equation of state is found for these solutions.

We also computed the first and second order fluctuations around a static string center of mass, using the string perturbation series approach [7] and its covariant versions [9, 26]. Up to second order, the mass-formula for arbitrary values of the curvature index (positive or negative) is identical to the wellknown flat spacetime mass-formula; all dependence on $K$ cancels out. 


\section{Conclusion}

We first studied the string propagation in the $2+1 \mathrm{BH}-\mathrm{AdS}$ background. We found the first and second order perturbations around the string center of mass as well as the mass formula, and compared with the ordinary black hole AdS spacetime and with the black string background as well. These results were then generalized to ordinary $D$-dimensional anti de Sitter spacetime. The classical string motion in anti de Sitter spacetime is stable in the sense that it is oscillatory in time with real frequencies and the string size and energy are bounded. Quantum mechanically, this reflects in the mass operator, which is well defined for any value of the wave number $n$, and arbitrary high mass states (and therefore an infinite number of states) can be constructed. This is to be contrasted with de Sitter spacetime, where string instabilities develop, in the sense that the string size and energy become unbounded for large de Sitter radius. For low mass states (the stable regime), the mass operator in de Sitter spacetime is given by Eq.(3.1) but with

$$
\Omega_{n}(\lambda)_{\mathrm{dS}}=\frac{2 n^{2}-m^{2} \alpha^{\prime} \lambda}{\sqrt{n^{2}-m^{2} \alpha^{\prime} \lambda}}
$$

Real mass solutions can be defined only up to some maximal mass of the order $m^{2} \alpha^{\prime} \approx 1 / \lambda$ [7]. For $\lambda<<1$, real mass solutions can be defined only for $N \leq N_{\max } \sim 0.15 / \lambda$ (where $N$ is the eigenvalue of the number operator) and therefore there exists a finite number of states only. These features of strings in de Sitter spacetime have been recently confirmed within a different (semi-classical) quantization approach based on exact circular string solutions [42].

The presence of a cosmological constant $\Lambda$ (positive or negative) increases considerably the number of levels of different eigenvalue of the mass operator (there is a splitting of levels) with respect to flat spacetime. That is, a non-zero cosmological constant decreases (although does not remove) the degeneracy of the string mass states, introducing a fine structure effect. For the low mass states the level spacing is approximately constant (up to corrections of the order $\lambda^{2}$ ). For the high mass states, the changes are more drastic and they depend crucially on the sign of $\Lambda$. A value $\Lambda<0$ causes the growing of the level spacing linearly with $N$ instead of being constant as in Minkowski space. Consequently, the density of states $\rho(m)$ grows with the exponential of $\sqrt{m}$ (instead of $m$ as in Minkowski space) discarding the 
existence of a Hagedorn temperature in AdS spacetime, and the possibility of a phase transition. In addition, another important feature of the high mass string spectrum in AdS spacetime is that it becomes independent of $\alpha^{\prime}$. The string scale for the high mass states is given by $|\Lambda|$, instead of $1 / \alpha^{\prime}$ for the low mass states.

We have found new classes of exact string solutions obtained either by the circular string ansatz, Eq.(2.16), or by the stationary string ansatz, Eq.(4.3), in a variety of curved backgrounds including Schwarzschild, de Sitter and anti de Sitter spacetimes. Many different types of solutions have been found: oscillating circular strings, closed stationary strings, infinitely long stationary strings, dynamical straight strings and multi-string solutions describing finitely or infinitely many stationary or dynamical strings. In all the cases we have obtained the exact solutions in terms of either elementary or elliptic functions. Furthermore, we have analyzed the physical properties (length, energy, pressure) of the string solutions.

Finally, we solved the equations of motion and constraints for circular strings in static Robertson-Walker spacetimes. We computed the equations of state and found that there exists a self-consistent solution to the Einstein equations in the case of positive spatial curvature. The solutions were quantized semi-classically using the stationary phase approximation method, and the resulting spectra were analyzed and discussed. We have also found all stationary string configurations in these spacetimes and we computed the corresponding physical quantities, string length, energy and pressure.

\section{Acknowledgements:}

A.L. Larsen is supported by the Danish Natural Science Research Council under grant No. 11-1231-1SE 


\section{References}

[1] H.J. de Vega and N. Sánchez, Phys. Rev. D42 (1990) 3969.

H.J. de Vega, M. Ramón-Medrano and N. Sánchez, Nucl. Phys. B374 (1992) 405.

[2] H.J. de Vega and N. Sánchez, Phys. Lett. B244 (1990) 215, Phys. Rev. Lett. 65C (1990) 1517, IJMP A7 (1992) 3043, Nucl. Phys. B317 (1989) 706 and ibid 731.

D. Amati and C. Klimcik, Phys. Lett. B210 (1988) 92.

M. Costa and H.J. de Vega, Ann. Phys. 211 (1991) 223 and ibid 235.

C. Loustó and N. Sánchez, Phys. Rev. D46 (1992) 4520.

[3] V.E. Zakharov and A.V. Mikhailov, JETP 47 (1979) 1017.

H. Eichenherr, in "Integrable Quantum Field Theories", ed. J. Hietarinta and C. Montonen (Springer, Berlin, 1982).

[4] H.J. de Vega and N. Sánchez, Phys. Rev. D47 (1993) 3394.

[5] I. Bars and K. Sfetsos, Mod. Phys. Lett. A7 (1992) 1091.

[6] H.J. de Vega, J.R. Mittelbrunn, M.R. Medrano and N. Sánchez, Phys. Lett. B232 (1994) 133 and "The Two-Dimensional Stringy Black Hole: a new Approach and a Pathology", PAR-LPTHE 93/14.

[7] H.J. de Vega and N. Sánchez, Phys. Lett. B197 (1987) 320.

[8] H.J. de Vega and N. Sánchez, Nucl. Phys. B299 (1988) 818.

[9] P.F. Mende, in "String Quantum Gravity and Physics at the Planck Energy Scale". Proceedings of the Erice Workshop held in June 1992, ed. N. Sánchez (World Scientific, 1993).

[10] A.L. Larsen and V.P. Frolov, Nucl. Phys. B414 (1994) 129.

[11] H.J. de Vega, A.V. Mikhailov and N. Sánchez, Teor. Mat. Fiz. 94 (1993) 232, Mod. Phys. Lett. A29 (1994) 2745.

[12] F. Combes, H.J. de Vega, A.V. Mikhailov and N. Sánchez, Phys. Rev. D50 (1994) 2754. 
[13] H.J. de Vega, A.L. Larsen and N. Sánchez, Nucl. Phys. B427 (1994) 643.

[14] I. Krichever, "Two-Dimensional Algebraic-Geometrical Operators with Self-Consistent Potentials", Landau Institute Preprint, March, 1994, to appear in Funct. Analisis and Appl.

[15] H.J. de Vega and N. Sánchez, Nucl. Phys. B309 (1988) 552 and ibid, 577.

[16] C.O. Loustó and N. Sánchez, Phys. Rev. D47 (1993) 4498.

[17] H.J. de Vega and I.L. Egusquiza, Phys. Rev. D50 (1994) 763.

[18] A.L. Larsen, Phys. Rev. D50 (1994) 2623.

[19] A.L. Larsen and M. Axenides, Phys. Lett. B318 (1993) 47.

[20] H.J. de Vega and N. Sánchez, Phys. Rev. D50 (1994) 7202.

[21] N. Sánchez and G. Veneziano, Nucl. Phys. B333 (1990) 253.

M. Gasperini, N. Sánchez and G. Veneziano, IJMP A6 (1991) 3853, Nucl. Phys. B364 (1991) 365.

[22] H.J. de Vega, M. Ramón-Medrano and N. Sánchez, Nucl. Phys. B374 (1992) 425.

[23] H.J. de Vega and N. Sánchez, Phys. Rev. D45 (1992) 2783.

M. Ramón-Medrano and N. Sánchez, Class. Quantum Grav. 10 (1993) 2007.

[24] N. Sánchez, Phys. Lett. B195 (1987) 160.

[25] H.J. de Vega, M. Ramón-Medrano and N. Sánchez, Nucl. Phys. B351 (1991) 277.

[26] A.L. Larsen and N. Sánchez, Phys. Rev. D50 (1994) 7493.

[27] A.L. Larsen and N. Sánchez, "Mass Spectrum of Strings in Anti de Sitter Spacetime", DEMIRM-Paris-94048, to appear in Phys. Rev. D. 
[28] A.L. Larsen and N. Sánchez, "New Classes of Exact Multi-String Solutions in Curved Spacetimes", DEMIRM-Paris-95003, to appear in Phys. Rev. D.

[29] A.L. Larsen and N. Sánchez, "The Effect of Spatial Curvature on the Classical and Quantum Strings", DEMIRM-Paris-95004.

[30] M. Bañados, C. Teitelboim and J. Zanelli, Phys. Rev. Lett. 69 (1992) 1849.

[31] G.T. Horowitz and D.L. Welch, Phys. Rev. Lett. 71 (1993) 328.

[32] N. Kaloper, Phys. Rev. D48 (1993) 2598.

[33] M. Bañados, M. Henneaux, C. Teitelboim and J. Zanelli, Phys. Rev. D48 (1993) 1506.

[34] C. Farina, J. Gamboa and A.J. Seguí-Santonja, Class. Quantum Grav. 10 (1993) L193.

N. Cruz, C. Martínez and L. Peña, Class. Quantum Grav. 11 (1994) 2731 .

[35] A. Achúcarro and M.E. Ortiz, Phys. Rev. D48 (1993) 3600.

D. Cangemi, M. Leblanc and R.B. Mann, Phys. Rev. D48 (1993) 3606.

[36] J. Horne and G.T. Horowitz, Nucl. Phys. B368 (1992) 444.

[37] T. Buscher, Phys. Lett. B201 (1988) 466, B194 (1987) 59.

[38] A. Vilenkin, Phys. Rev. D24 (1981) 2082.

[39] H.J. de Vega and N. Sánchez, Int. J. Mod. Phys. A7 (1992) 3043.

[40] V.P. Frolov, V.D. Skarzhinsky, A.I. Zelnikov and O. Heinrich, Phys. Lett. B224 (1989) 255.

[41] R. Dashen, B. Hasslacher and A. Neveu, Phys. Rev. D11 (1975) 3424.

[42] H.J. de Vega, A.L. Larsen and N. Sánchez, "Semi-Classical Quantization of Circular Strings in de Sitter and anti de Sitter Spacetimes", DEMIRM-Paris-94049, submitted to Phys. Rev. D. 
[43] A.L. Larsen, "Stable and Unstable Circular Strings in Inflationary Universes", NORDITA-94/14-P, to appear in Phys. Rev. D.

[44] A.L. Larsen, Nucl. Phys. B412 (1994) 372. 


\section{Figure Captions}

Figure 1. The potential $V(r)$, Eq.(2.21), for a circular string in the $2+1$ $\mathrm{BH}$-AdS spacetime. In (a) we have $J^{2}>4 E^{2}$ and a barrier between the inner horizon and $r=0$, while (b) represents a case where $J^{2}<4 E^{2}$ and a string will always fall into $r=0$. The static limit is $r$ erg $=l$.

Figure 2. The potential $V(r)$, Eq.(2.18), for a circular string in the equatorial plane of the four $3+1$ dimensional spacetimes: (a) Minkowski (Min), (b) Schwarzschild black hole (S), (c) anti de Sitter (AdS), and (d) Schwarzschild anti de Sitter space (S-AdS).

Figure 3. The $(N, M)=(5,1)$ multi-string solution in anti de Sitter spacetime. The $(N, M)$ multi-string solutions describe $N$ stationary strings with $M$ windings in the Azimuthal angle $\phi$, in anti de Sitter spacetime.

Figure 4. The $(N, M)=(3,2)$ stationary string solution inside the horizon of de Sitter spacetime. Besides the circular string, this is the simplest stationary closed string configuration in de Sitter spacetime.

Figure 5. Schematic representation of the time evolution of the $(N, M)=$ $(5,1)$ dynamical multi-string solution, outside the horizon of de Sitter spacetime. Only one of the 5 strings is shown; the others are obtained by rotating the figure by the angles $2 \pi / 5,4 \pi / 5,6 \pi / 5$ and $8 \pi / 5$. During the "scattering" at the horizon, the string collapses to a point and re-expands.

Figure 6. The dynamical straight string inside the horizon of the Schwarzschild black hole. The string infalls non-radially towards the singularity with indefinetely growing length.

Figure 7. The potential $V(r)$ introduced in Eqs.(5.3) for a circular string in the static Robertson-Walker spacetimes: (a) flat $(K=0)$, (b) closed $\left(K>0\right.$ and $\left.b K \alpha^{\prime 2} \leq 1\right)$, (c) closed $\left(K>0\right.$ and $\left.b K \alpha^{\prime 2}>1\right)$, (d) hyperbolic $(K<0)$.

Figure 8. Parametric plot of $K \alpha^{\prime} W$ as a function of $K \alpha^{\prime 2} m^{2}$ in the three cases: (a) $K>0, b K \alpha^{2} \leq 1$ (strings oscillating on one hemisphere, only 
finitely many states), (b) $K>0, b K \alpha^{\prime 2}>1$ (strings oscillating on the full hypersphere, infinitely many states), (c) $K<0$ (strings oscillating on the hyperboloid, infinitely many states).

Figure 9. The potential $V(r)$ introduced in Eqs.(5.10) for a stationary string in the static Robertson-Walker spacetimes: (a) flat $(K=0)$, (b) closed $\left(K>0\right.$ and $\left.K L^{2} \leq 1\right)$, (c) hyperbolic $(K<0)$. 


\section{Table Captions}

Table 1. String motion described by the string perturbation series approach in the $2+1 \mathrm{BH}-\mathrm{AdS}$, ordinary black hole-AdS, de Sitter (dS) and black string backgrounds.

Table 2. Circular exact string solutions in the indicated backgrounds. $S(\tau)$ is the invariant string size.

Table 3. Characteristic features of the quantum string mass spectrum in anti de Sitter (AdS) and de Sitter (dS) spacetimes.

Table 4. Short summary of the features of the string solutions found in this section. In anti de Sitter spacetime and outside the horizon of de Sitter and $2+1$ BH-AdS spacetimes, the solutions describe a finite number of strings provided a condition of the form $N \Delta \phi=2 \pi M$ is fulfilled, where $\Delta \phi$ is the angle betwen the "arms" of the string and $(N, M)$ are integers.

Table 5. Classical circular strings in the static Robertson-Walker spacetimes. Notice that a self-consistent solution to the Einstein equations, with the string back-reaction included, can be obtained only for $K>0$.

Table 6. Semi-classical quantization of the circular strings in the static Robertson-Walker spacetimes. Notice in particular the different behaviour of the high mass spectrum of strings in the three cases.

Table 7. Stationary strings in the static Robertson-Walker spacetimes. Notice that the pressure densities are always negative in all three cases. 
This figure "fig1-1.png" is available in "png" format from: http://arxiv.org/ps/hep-th/9504007v1 
This figure "fig2-1.png" is available in "png" format from: http://arxiv.org/ps/hep-th/9504007v1 
This figure "fig1-2.png" is available in "png" format from: http://arxiv.org/ps/hep-th/9504007v1 
This figure "fig2-2.png" is available in "png" format from: http://arxiv.org/ps/hep-th/9504007v1 
This figure "fig1-3.png" is available in "png" format from: http://arxiv.org/ps/hep-th/9504007v1 
This figure "fig2-3.png" is available in "png" format from: http://arxiv.org/ps/hep-th/9504007v1 
This figure "fig1-4.png" is available in "png" format from: http://arxiv.org/ps/hep-th/9504007v1 
This figure "fig2-4.png" is available in "png" format from: http://arxiv.org/ps/hep-th/9504007v1 
This figure "fig1-5.png" is available in "png" format from: http://arxiv.org/ps/hep-th/9504007v1 
This figure "fig2-5.png" is available in "png" format from: http://arxiv.org/ps/hep-th/9504007v1 
This figure "fig1-6.png" is available in "png" format from: http://arxiv.org/ps/hep-th/9504007v1 
This figure "fig2-6.png" is available in "png" format from: http://arxiv.org/ps/hep-th/9504007v1 
This figure "fig1-7.png" is available in "png" format from: http://arxiv.org/ps/hep-th/9504007v1 
This figure "fig2-7.png" is available in "png" format from: http://arxiv.org/ps/hep-th/9504007v1 
This figure "fig1-8.png" is available in "png" format from: http://arxiv.org/ps/hep-th/9504007v1 
This figure "fig1-9.png" is available in "png" format from: http://arxiv.org/ps/hep-th/9504007v1 